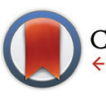

CrossMark click for updates

Cite this: Org. Biomol. Chem., 2016, 14, 10547

Received 28th July 2016, Accepted 21st September 2016

DOI: 10.1039/c6ob01618a

www.rsc.org/obc

\title{
Fluorinated diazoalkanes - a versatile class of reagents for the synthesis of fluorinated compounds
}

\begin{abstract}
Lucas Mertens and Rene M. Koenigs*
Although diazoalkanes find regular application in organic synthesis, the synthesis and application of their fluorinated homologues was long neglected. First described in 1943, trifluorodiazoethane found its way into the organic synthesis repertoire only in the past decade for atom-economical and practical synthesis of fluorinated building blocks and currently emerges as a versatile reagent. The synthetic applicability of this reagent is currently being investigated in great detail and many interesting new reactivities, and procedures for the synthesis of fluoroalkyl-substituted building blocks have been developed. In this context, a range of new fluoroalkyl substituted diazoalkanes have been introduced in the past few years, ranging from perfluorinated diazoalkanes towards highly reactive difluorodiazoethane which was first described in 2015. This tutorial review covers historic milestones of fluoroalkyl substituted diazoalkanes and highlights recent examples which underscore their vast potential to the synthesis of fluorinated compounds.
\end{abstract}

\section{Introduction}

Diazomethane, first described in 1894 by von Pechmann, represents the shortest purely aliphatic homologues of alkyl diazo compounds. ${ }^{1}$ Since then the synthetic application of diazomethane has been investigated to a great extent. ${ }^{2,3}$ More importantly, the use of state-of-the-art synthesis technologies such as continuous-flow chemistry has made significant progress with respect to lab safety and scalability. ${ }^{4-6}$ Regarding the toxicity of diazomethane, von Pechmann already stated in his seminal report:

“...besitzt es höchst giftige Eigenschaften, welche zunächst Athemnoth [...] hervorrufen und das Arbeiten damit äusserst unangenehm machen."

"...it possesses highly toxic properties, which evoke respiratory depression [...] and make handling particularly unpleasant."

Although chemical synthesis using diazomethane requires strict safety regulations, applications of the smallest diazoalkane have become indispensable for modern organic synthesis. Similarly, substituted diazomethanes have found widespread application across the whole field of organic synthesis.

Interestingly, fluorinated diazoalkanes 1-4 (Fig. 1) are investigated to a much lower extent, although they represent a very practical and versatile class of reagents that enable chemists to quickly introduce fluorinated side chains from simple and readily available precursors.

RWTH Aachen University, Institute of Organic Chemistry, Landoltweg 1, D-52074 Aachen, Germany. E-mail: rene.koenigs@rwth-aachen.de

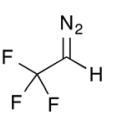

1

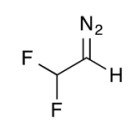

2

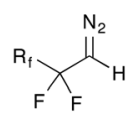

3

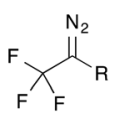

4
Fig. 1 Fluoroalkyl substituted diazoalkanes.

Trifluorodiazoethane (1) is probably the best-studied fluorinated diazoalkane. First described in 1943 by Gilman and Jones through reaction of trifluoroethylamine and nitrous acid, $^{7}$ this reagent only found regular application in organic synthesis in the past decade as a versatile reagent yielding versatile fluorinated building blocks in a very efficient and atomeconomical fashion.

Application of fluorinated diazoalkanes is of particular importance for chemists from all fields. Long perfluoroalkyl groups are of high interest in the development of materials, such as OLEDs. ${ }^{8}$ The short-chain analogues are of high importance to the pharmaceutical and agrochemical industry and allow the efficient construction of small fluorinated building blocks that find regular application in discovery programs. ${ }^{9,10}$

This tutorial review will concentrate on the organic chemistry of fluoroalkyl-substituted diazoalkanes (1-3), covering the range from historic milestones towards a detailed discussion of the current synthetic methodology. Technical aspects, such as the preparation of diazo compounds will be discussed as part of the synthesis application described herein. 


\section{Early applications}

In their seminal report Gilman and Jones reported on the deca-gram scale synthesis of trifluoroethylamine hydrochloride $(\mathbf{8} \cdot \mathbf{H C l})$ from commercially available sodium trifluorocarboxylate (5). The free base (8) readily reacts in the presence of sulphuric acid with sodium nitrite in water under the formation of trifluorodiazoethane (1) that can be distilled as an ethereal solution with no apparent decomposition and $65-67 \%$ yield on the multi-gram scale (Scheme 1). Gilman and Jones also reported the synthesis of the neat reagent, which can be distilled at a boiling point of 13 to $13.5^{\circ} \mathrm{C}$. From a synthetic perspective, the reaction with iodine is the only application described within this manuscript. ${ }^{7}$

Although Gilman and Jones reported on the synthesis of trifluorodiazoethane in detail, synthetic applications of this reagent were not reported until 1964. Fields and Haszeldine investigated decomposition and polymerization reactions of trifluorodiazoethane and heptafluoropropyl diazomethane, yet no applications for the synthesis of small molecules have been described within this manuscript. ${ }^{11}$ It should be noted that the authors describe serious risk hazards and severe explosions when working with these reagents.

In further reports Atherton and Fields reported proof of principle studies on cycloaddition reactions of trifluorodiazoethane with olefins yielding cyclopropanes ${ }^{12}(9)$ or pyrazolines (10 and 11) (Fig. 2), ${ }^{13}$ and on insertion reactions providing trifluoroethyl substituted alkanes, silanes, ethers and amines. ${ }^{14}$

Branched fluoroalkyl-substituted diazo compounds were investigated by Stone and co-workers and their application as ligands in transition metal complexes. ${ }^{15}$

Meese reported the reaction of trifluorodiazoethane with organic acids in 1984. Interestingly, no reaction was observed upon treatment of trifluorodiazoethane with carboxylic acids,

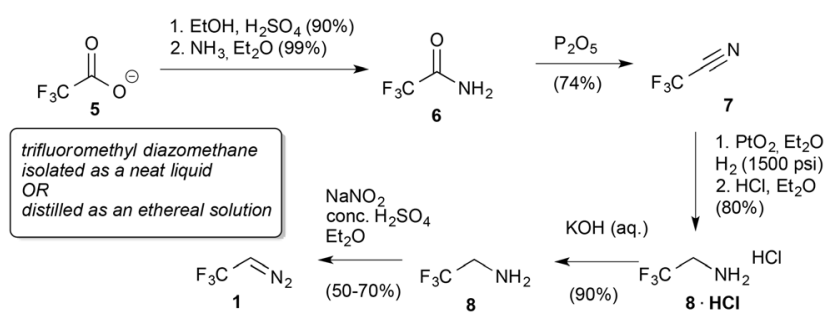

Scheme 1 Synthesis of trifluoroethyl amine and trifluorodiazoethane by Gilman and Jones.
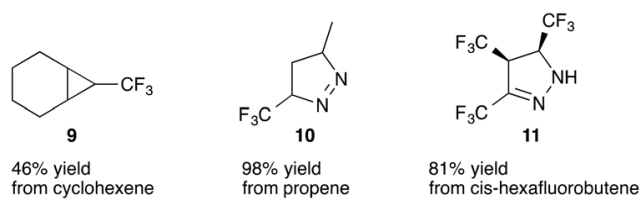

Fig. 2 Reaction products obtained from trifluorodiazoethane.
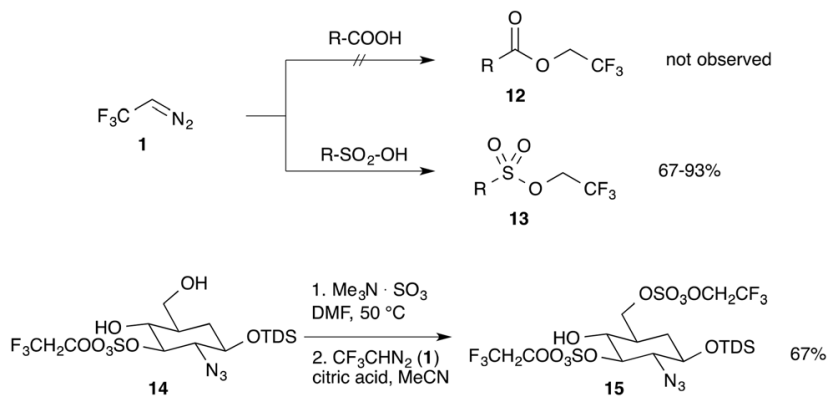

Scheme 2 Reaction of trifluorodiazoethane with carboxylic acids and sulfonic acids.

whereas the reaction with sulfonic acids yielded the corresponding sulfonates (13) in high yields. This is a remarkable observation and it highlights the unique nature of this reagent, as the typical esterification reaction of diazo compounds with carboxylic acids, such as acetic acid or benzoic acids is not taking place. ${ }^{16}$ This very unique transformation was applied to the preparation of sulpho protected glycosaminoglycans (15) by Linhardt and co-workers (Scheme 2). ${ }^{17}$

In further investigations, the reaction of trifluorodiazoethane with alcohols ${ }^{18}$ glyoxylate,${ }^{19}$ chloral $^{20}$ and aldehydes $^{21}$ was studied. Additionally, trifluorodiazoethane was applied in the synthesis of pyrazoles ${ }^{22}$ and in ring expansion reactions to yield 7 -membered rings. ${ }^{23}$ Although all of these transformations represent early examples of the applicability of this reagent to organic synthesis, they have in common that only a very limited substrate scope has been investigated.

At this point it should be noted that the correct IUPAC name of $\mathbf{1}$ is 2,2,2-trifluoro diazoethane. In the recent literature, the term trifluoromethyl diazomethane has been introduced, and it is important to know that both terms are used in the current literature.

The challenge when working with fluoroalkyl-substituted diazoalkanes is the significantly reduced nucleophilicity at the diazo-carbon atom, due to the strongly electron-withdrawing nature of the fluoroalkyl group. This review will outline the latest strategies and methods that have been developed to utilize this class of reagents in organic synthesis.

\section{Applications of trifluorodiazoethane}

\subsection{Cyclopropanation reactions}

Undoubtedly, cyclopropanation reactions are one of the most important synthetic applications of diazo compounds. ${ }^{24-26}$ After the very first report on trifluoromethyl cyclopropanes by Atherton and Fields in 1968, it took almost 40 years until Simonneaux and co-workers reported on iron(III) catalysed enantioselective cyclopropanation using trifluorodiazoethane with a broad range of different styrenes. A chiral homogeneous iron porphyrin complex 18 (Fig. 3) served for chiral induction. 


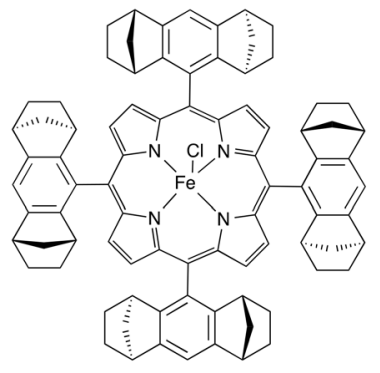

18

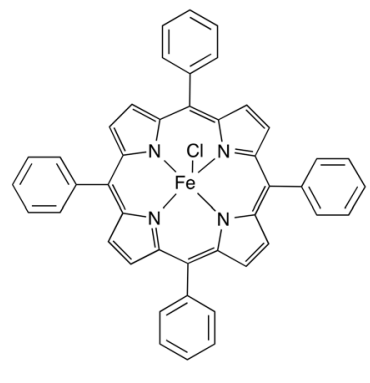

22
Fig. 3 Iron catalysts used for cyclopropanation reactions with trifluorodiazoethane.

Although the authors observed high diastereoselectivity, only moderate enantiomeric excess $(17-75 \%$ ee) and limited yield (24-96\%) could be obtained (Scheme 3). Still, this report marks the beginning of modern trifluorodiazoethane chemistry. ${ }^{27}$

A critical drawback of the method described by Simonneaux and co-workers is the preparation of the diazo compound. Similarly to the early reports on trifluorodiazoethane, Simonneaux and co-workers prepared the diazo compound in a neat form by cold distillation for subsequent cyclopropanation reactions, which is far from being practical.

In 2007, Davies and co-workers achieved a remarkable improvement in cyclopropanation reactions using 2,2,2-trifluoroethane hydrazones (19) that can be readily oxidized to the diazoalkanes with $\mathrm{MnO}_{2}$. Application of 1-aryl-2,2,2-trifluorodiazoethanes (4) and chiral Rh(II) catalysts proved to be superior in cyclopropanation reactions with styrenes and the desired trifluoromethyl substituted cyclopropanes (20) could be obtained with high diastereoselectivity (>94\%), high enantiomeric excess $(88-94 \%$ ee) and good chemical yield (61-76\%). However, aliphatic olefins provided the desired reaction products only with significantly reduced yield (Scheme 4). ${ }^{28}$

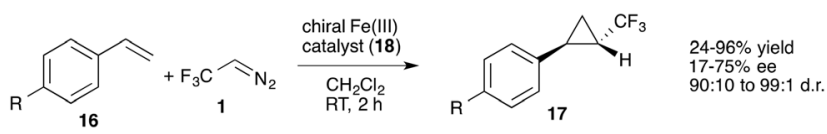

Scheme 3 Iron catalysed enantioselective cyclopropanation by Simonneaux and co-workers.

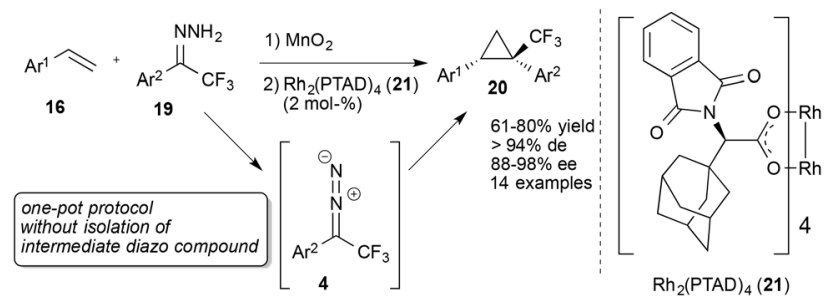

Scheme 4 Rhodium(॥) catalysed enantioselective cyclopropanation of styrenes.
Shortly after these reports, Komarov and co-workers reported on general $\mathrm{Rh}$ (II) and $\mathrm{Cu}$ (I) catalysed cyclopropanation reactions using trifluorodiazoethane. For the first time, Komarov and co-workers were able to demonstrate dihydropyran, enol ethers, dehydro amino acids and cyclohexene to be suitable substrates for cyclopropanation reactions using trifluorodiazoethane as the carbene precursor. ${ }^{29}$ Additionally, trifluoromethyl substituted proline analogues have been reported. $^{30}$

In 2010, Carreira and co-workers achieved a remarkable break-through in cyclopropanation reactions using trifluorodiazoethane. Application of an achiral Fe(III) porphyrin catalyst 22 (Fig. 3) and in situ generation of trifluorodiazoethane in water were key to this method. This result is remarkable as it is the first example describing the utilization of trifluoroethyl amine and the direct reaction of in situ generated fluorinated diazoalkane with an olefin, which significantly facilitates the synthetic applicability of this versatile reagent (Scheme 5 ). ${ }^{31}$

Carreira and co-workers reported on extensions of this work in 2011. Application of dienes and enynes in this cyclopropanation reaction furnished valuable vinyl- and alkynyl-substituted cyclopropanes. Carreira and co-workers used a similar protocol as in their initial report and were able to obtain these building blocks in high yield with excellent diastereoselectivities. ${ }^{32}$

Using a chiral salen-based Co(II)-catalyst (25) highly diastereoselective and enantioselective cyclopropanation reactions of styrenes on water could be achieved. Interestingly additional organic co-solvents resulted in reduced conversion to the desired cyclopropane (Scheme 5). ${ }^{33}$

Moreover, Carreira and co-workers reported on Rh(II) catalysed cyclopropanation reactions. Different aliphatic and aromatic alkynes could be converted to the corresponding cyclopropene with high yield using $\mathrm{Rh}_{2} \mathrm{esp}_{2}$ as a catalyst (Scheme 12). ${ }^{34}$

These ground breaking proof of principle reports on the application of trifluorodiazoethane in organic synthesis set the stage for a rich chemistry that followed in the next years.

Further applications of trifluorodiazoethane in cyclopropanation reactions concentrated on extensions of the substrate scope towards electron-rich olefins. Mykhailiuk reported $[x .1 .0]$-bicycles $(x=2,3,4)$ that are versatile building blocks for
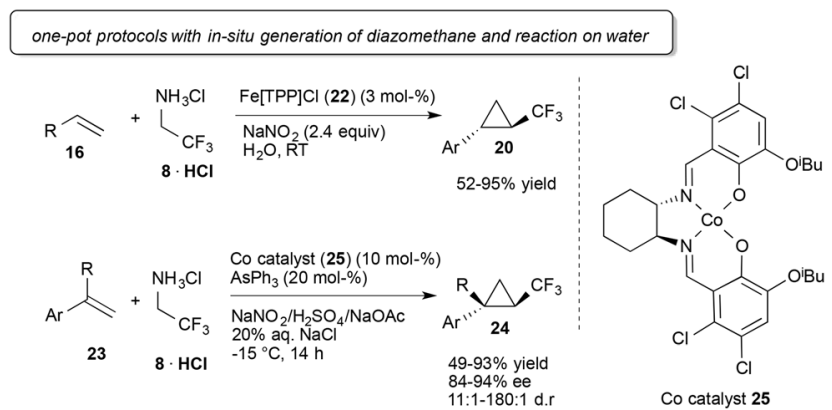

Scheme 5 Cyclopropanation reactions on water. 


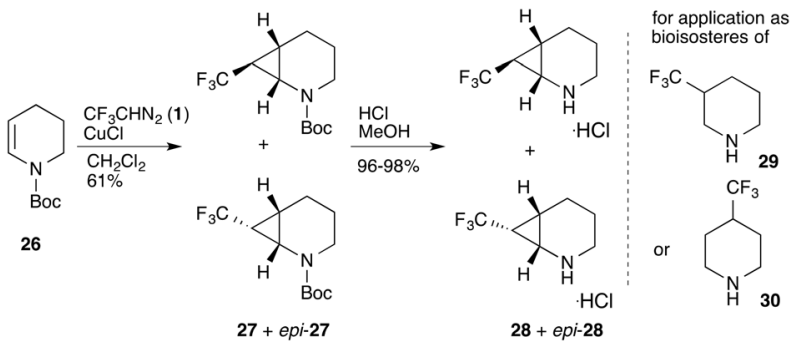

Scheme 6 Cyclopropanation reactions with enamides.

applications in pharmaceutical and agrochemical research programs. In particular, the authors report on $\mathrm{Cu}(\mathrm{I})$ catalysed cyclopropanation reactions using cyclic enamides which provide direct access to building blocks that can be used for bioisosteric replacement of substituted trifluoromethyl substituted cyclohexanes (Scheme 6). ${ }^{35}$

The reaction sequence of $[2+3]$ cycloaddition and subsequent cycloreversion under extrusion of nitrogen provides an alternative and efficient pathway towards cyclopropanes, the advantage being that this approach does not require the use of expensive metal catalysts.

Mykhailiuk and co-workers first reported on this approach for the synthesis of trifluoromethyl cyclopropanes. Using maleimides, which readily react in $[2+3]$ cycloaddition, the authors first prepared the corresponding pyrazolines, which then release nitrogen upon heating to $150{ }^{\circ} \mathrm{C}$ (Scheme 7). ${ }^{36}$

$\mathrm{Lu}$, Xiao and co-workers reported the application of oxindoles in this particular transformation. In a metal-free transformation, trifluorodiazoethane was generated in situ from trifluoro ethylamine hydrochloride and then added to oxoindolin 31 resulting in spirocyclic pyrazoline $\mathbf{3 2}$ with high yield. In a next step this pyrazoline undergoes cycloreversion under thermal conditions yielding the desired trifluoromethyl substituted cyclopropane $\mathbf{3 3}$ in good yield and excellent diastereoselectivity. ${ }^{37}$

In 2015, Ma and coworkers reported an interesting cyclopropanation reaction of trifluorodiazoethane and azlactones. While typical cyclopropanation catalysts like CuOTf, FeTPPCl or $\mathrm{Rh}_{2} \mathrm{esp}_{2}$ proved inefficient, heating of the reaction mixture without a catalyst in the presence of brine afforded the desired cyclopropane in good yield and excellent diastereoselectivity. According to the authors, brine stabilizes the diazoalkane and thus leads to increased yield. The authors assume that this

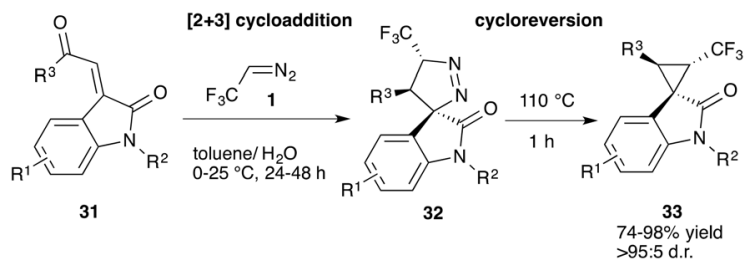

Scheme 7 Cycloaddition-cycloreversion for the synthesis cyclopropanes.

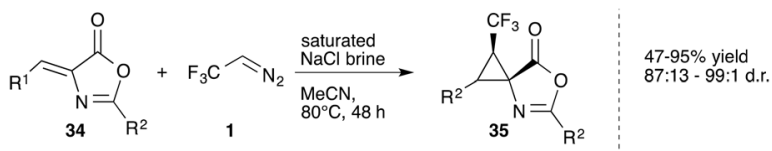

Scheme 8 Brine stabilized synthesis of cyclopropanes.

cyclopropanation may proceed either by $[2+1]$ cycloaddition of the carbene or alternatively by an initial $[2+3]$ cycloaddition yielding a pyrazole that liberates nitrogen under the formation of the cyclopropane (Scheme 8). ${ }^{38}$

An extension of this work was reported by Ma and Cahard towards benzofuran-2(3H)-ones. ${ }^{39}$

Trifluoromethyl-substituted cyclopropanes find application as TRPV1 antagonists. Duncton and co-workers reported on the cyclopropanation reaction of vinyl dibutyl borate with trifluorodiazoethane and subsequent application of this boronic ester in the preparation of the corresponding trifluoroborate potassium salt. This salt can be coupled to different aromatic esters and serves as an entry into highly potent human TRPV1 antagonists (Fig. 4$)^{40}$

\subsection{Dipolar cycloadditions}

As outlined in the last paragraph on cyclopropanation reactions, substantial progress has been made with regard to dipolar cycloaddition reactions with electron-poor olefins. ${ }^{41}$ This section will focus on applications of $[2+3]$ cycloaddition reactions with the aim of preparing pyrazoles and pyrazolines.

$[2+3]$ cycloaddition reactions are one of the most commonly applied reactions of diazo compounds. Their dipolar nature makes them formidable substrates for the efficient synthesis of pyrazoles and pyrazolines, both being highly desired heterocycles for research in pharmaceutical and agrochemical industries.

Fluoroalkyl-substituted pyrazoles undoubtedly belong to one of the most important classes of heterocycles that find many applications in industrial research. Among the most prominent examples of fluoroalkyl substituted pyrazoles are COX inhibitors, such as celecoxib (38), mavacoxib (40) or deracoxib (39). ${ }^{42,43}$ Similarly, this class of compounds finds regular application in Factor Xa discovery programs, which led to the discovery of razaxaban (41) (Fig. 5). ${ }^{44}$ Typically, the preparation of these heterocycles requires multi-step synthesis and suffers from regioselectivity problems during preparation.

Initial reports by Fields and co-workers towards $[2+3]$ cycloaddition reactions of trifluorodiazoethane remained

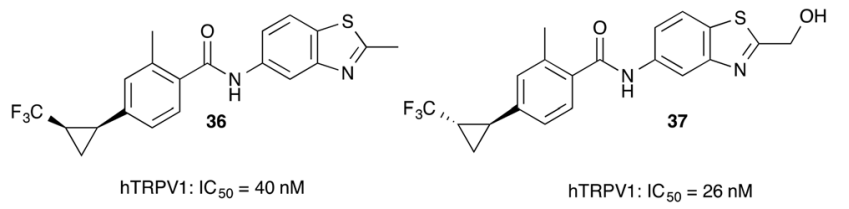

Fig. 4 TRPV1 antagonists reported by Duncan and co-workers. 

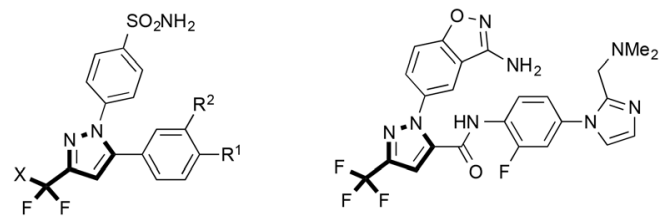

Celecoxib $38\left(X=H, R^{1}=M e R^{2}=H\right)$

Razaxaban 41

Deracoxib $39\left(X=F, R^{1}=F, R^{2}=O M e\right)$

Mavacoxib $40\left(X=F, R^{1}=F, R^{2}=H\right)$

Fig. 5 Fluoroalkyl substituted pyrazoles.

unpractical due to long and harsh reaction conditions and limited substrate scope. ${ }^{13,22}$

Recently, Ma and co-workers reported significant progress with regard to this versatile transformation. In their report on dipolar cycloaddition reactions the authors investigated the effect of Lewis acids in the reaction of trifluorodiazoethane with terminal alkynes (42). Ag(I) salts proved to be superior for this transformation and the highly interesting trifluoromethylated pyrazoles (43) could be obtained in a single reaction step with high chemical yield with exquisite regioselectivity (Scheme 9). ${ }^{45}$

In an additional report on [2+3] cycloaddition Mykhailiuk reported an important improvement in the practical study when using trifluorodiazoethane. ${ }^{46}$ Inspired by the work of Carreira and co-workers, ${ }^{31}$ the authors described the synthesis and direct synthetic application of this reagent using a biphasic aqueous/organic solvent mixture. The advantage of the new protocols, described by Carreira and Mykhailiuk, is that there is no need for purification of the diazo compound and that hazards can be minimized as the diazo compound is directly consumed.

With regard to the preparation of the diazo compound, it should be noted that application of trifluoroethyl amine hydrochloride and sodium nitrite serves as a source for the on-water synthesis of trifluorodiazoethane and subsequent reactions can be either conducted on water, in a bi-phasic mixture by taking the organic layer and followed by addition to the reaction solution. Using this methodology, Mykhailiuk and coworkers were able to demonstrate that a broad range of olefins and alkynes bearing electron-withdrawing substituents could be converted to the corresponding pyrazoles with excellent yield (Scheme 10).

Koenigs and co-workers further investigated this transformation using a continuous-flow protocol, which relies on the preparation of trifluorodiazoethane from the free base trifluoroethyl amine and tert butyl nitrite in the presence of catalytic amounts of acetic acid. This protocol thus serves as

$$
\begin{aligned}
& { }_{42}=+{ }_{\mathrm{F}_{3} \mathrm{C}} \overbrace{\mathrm{N}_{2}} \underset{\mathrm{DMF}, 45^{\circ} \mathrm{C}, 5 \mathrm{~h}}{\mathrm{Ag}_{2} \mathrm{O}, \mathrm{NaOAC}} \\
& 51-95 \% \text { yield }
\end{aligned}
$$

Scheme 9 Silver catalysed synthesis of pyrazoles.

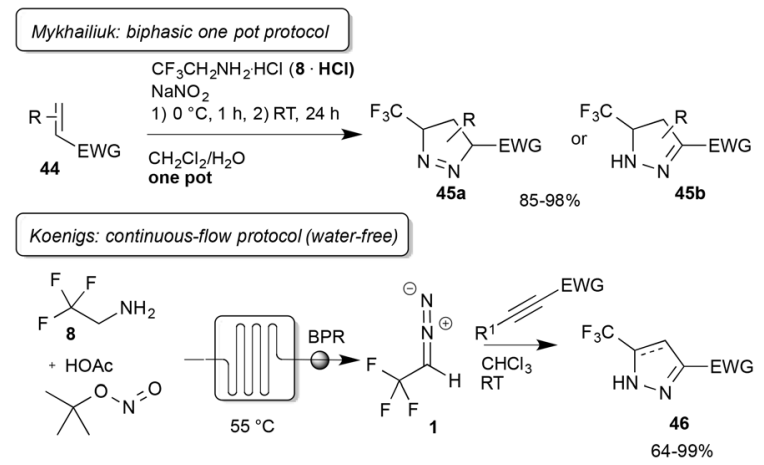

Scheme 10 Synthesis of pyrazolines by Mykhailiuk and Koenigs.

an option for the water-free preparation of this important reagent and allows an operationally simple handling of toxic diazo compounds (Scheme 10). ${ }^{47}$

$\mathrm{Ma}$ and co-workers recently reported an extension of this protocol towards the reaction of trifluorodiazoethane with allenes (47a and $\mathbf{4 7 b})^{48}$ and to benzanellated pyrazoles (48) through reaction of trifluorodiazoethane with arynes. ${ }^{49}$

$[2+3]$ cycloaddition reactions not only provide convenient access towards pyrazoles and pyrazolines, but can also be applied to the synthesis of a range of different other 5-membered heterocycles. Carreira and co-workers were first to report trifluorodiazoethane in the synthesis of trifluoromethylated benzofurans (49). ${ }^{50}$ Molander and co-workers reported on the synthesis of isoxazolidines $(\mathbf{5 0})^{51}$ and $\mathrm{Ma}$ and co-workers were able to demonstrate the applicability of trifluorodiazoethane in the synthesis of oxazolines (51) (Fig. 6). ${ }^{52}$

From a mechanistic perspective, these three reports differ significantly. The reaction of salicylaldehydes with trifluorodiazoethanes proceeds through Lewis-acid catalysed homologation of the aldehyde and subsequent hemi-acetal formation and dehydration to yield trifluoromethylated benzofurans. This homologation reaction will be discussed in detail in a separate paragraph of this review.

Contrarily, the synthesis of isoxazoles and oxazoles proceeds via $[2+3]$ cycloaddition reactions. Here, we discuss this
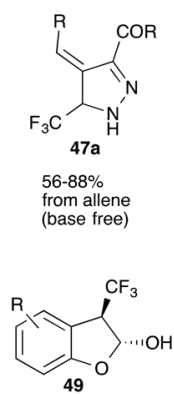

$54-72 \%$

from salicylaldehyde $\mathrm{BF}_{3} \cdot \mathrm{Et}_{2} \mathrm{O}$ catalytic amounts of

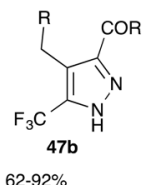

$62-92 \%$
from allene (in the presence of $\mathrm{NEt}_{3}$ )

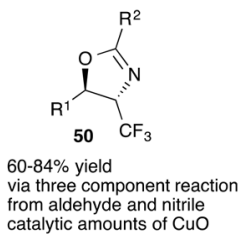

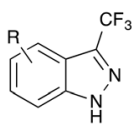

48

$60-85 \%$ via aryne<smiles>[R]C1ON([14CH3])C(C(F)(F)[18F])C1[R]</smiles>

via three component reaction from nitroso compound and
Fig. 6 Trifluoromethyl substituted heterocycles prepared with trifluorodiazoethane. 

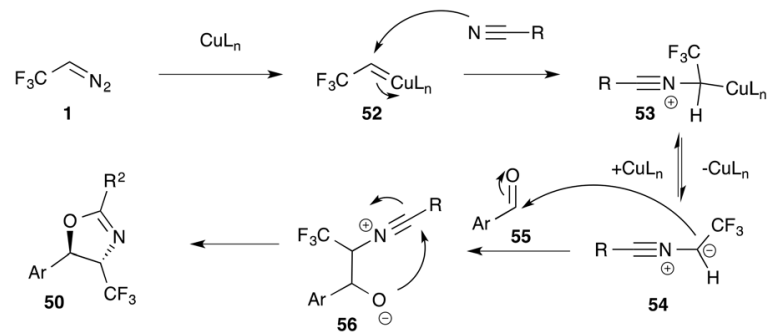

Scheme 11 Mechanism of the oxazol synthesis.

transformation for the synthesis of oxazoles exemplarily. Coordination to $\mathrm{Cu}(\mathrm{I})$ furnishes electrophilic intermediate 52, that undergoes reaction with a nitrile and formation of a 1,2dipole 54, which last undergoes stepwise [2+3] cycloaddition with an aldehyde (Scheme 11).

\subsection{Insertion reactions}

Insertion reactions are another very important application of diazo compounds. In the past a great variety of different catalytic $\mathrm{C}-\mathrm{H}$ and heteroatom-H insertion reactions has been developed allowing efficient and sustainable synthesis of important building blocks. ${ }^{53-55}$

This section will outline the achievements that have been made in the past few years utilizing fluoroalkyl-substituted diazoalkanes. These transformations are particularly important as they provide direct access towards fluorinated small molecules with exquisite synthetic efficiency.

In 2012, Ma and co-workers reported a protocol that is complementary to the cyclopropanation reaction described by Carreira and co-workers. Using catalytic amounts of $\mathrm{Cu}(\mathrm{I})$, $\mathrm{Ma}$ and co-workers have been able to demonstrate that terminal alkynes undergo $\mathrm{C}-\mathrm{H}$ insertion reactions yielding trifluoroethyl-substituted alkynes (58). ${ }^{56}$ This is remarkable as $\mathrm{Rh}_{2} \mathrm{esp}_{2}$ provides cyclopropanes $(\mathbf{5 9})^{34}$ and is an interesting example of catalyst controlled transformations (Scheme 12).

In addition to previously described $\mathrm{C}-\mathrm{H}$ insertion reactions, Wang and co-workers investigated $\mathrm{Ag}(\mathrm{I})$ catalysed insertion reactions into $\mathrm{N}-\mathrm{H}$ bonds. ${ }^{57}$ While $\mathrm{Ag}(\mathrm{I})$ catalysed insertion reactions into the $\mathrm{N}-\mathrm{H}$ bond of anilines yielded trifluoroethylsubstituted secondary amines (61), the $\mathrm{Ag}(\mathrm{I})$ catalysed reaction with benzamides gave $O$-trifluoroethyl imidates selectively. Competitive experiments with olefins did not provide cyclopropane products, which the authors attribute to the following postulated reaction mechanism (Scheme 13).

Recently, Gouverneur and co-workers reported further extension of the insertion reactions of trifluorodiazoethane

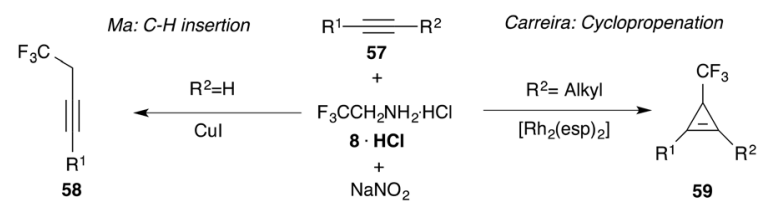

Scheme 12 Catalyst controlled reactions of trifluorodiazoethane.

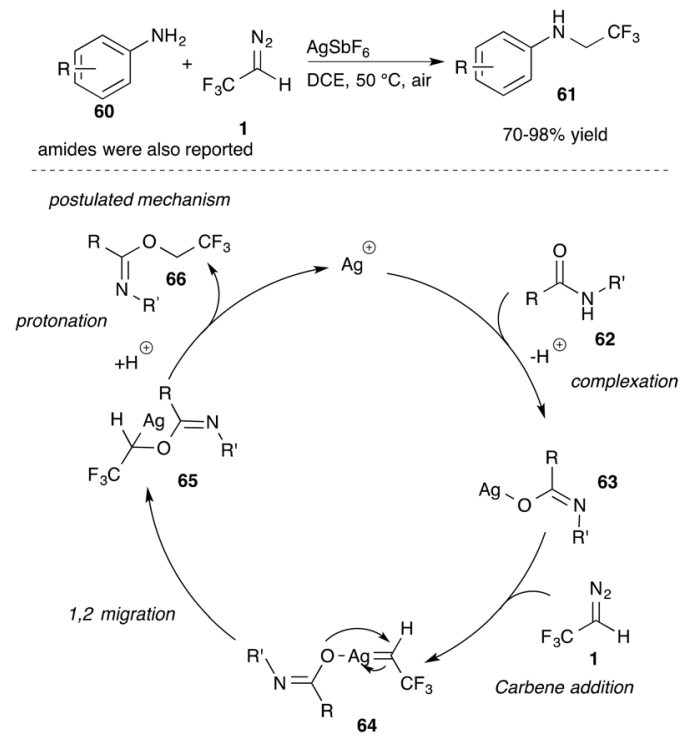

Scheme 13 Insertion reaction into the $\mathrm{N}-\mathrm{H}$ bond.

into heteroatom- $\mathrm{H}$ bonds. Application of a $\mathrm{Cu}(\mathrm{I})$ catalyst provided the insertion product into $\mathrm{B}-\mathrm{H}, \mathrm{Si}-\mathrm{H}, \mathrm{S}-\mathrm{H}, \mathrm{P}-\mathrm{H}$ and $\mathrm{N}-\mathrm{H}$ bonds (Scheme 14). ${ }^{58}$

Molander and co-workers reported further applications of trifluorodiazoethane in insertion reactions. Reaction of this diazo compound with aromatic boronic acids/esters or trifluoroborate potassium salts 69 provides the corresponding C-B bond insertion products (72) with excellent yield (Scheme 15). ${ }^{59}$ This reaction is particularly useful, as valuable trifluoromethyl substituted boron compounds can be easily obtained, which are useful intermediates for further functionalization, e.g. in Suzuki couplings, addition reactions to olefins or radical chemistry to name a few. In another report, Molander reported on the double homologation reaction of boroxines. For this transformation pinacol is a crucial additive to allow the second homologation step to proceed. ${ }^{60}$

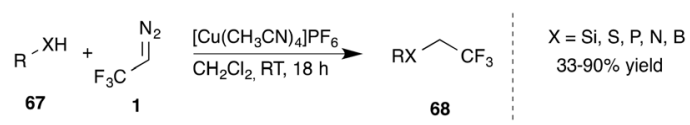

Scheme 14 Insertion into different $\mathrm{X}-\mathrm{H}$ bonds by Gourverneur and co-workers.

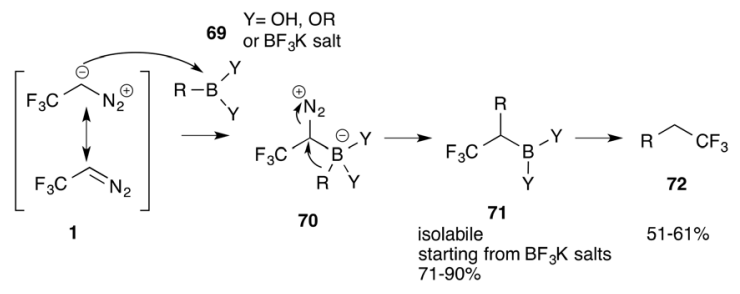

Scheme 15 Mechanism of the homologation reaction of boronic acids. 


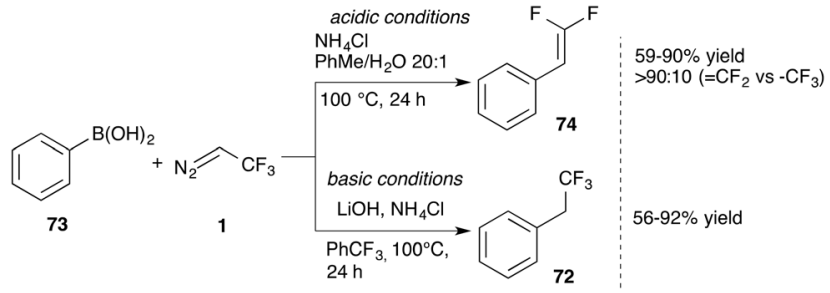

Scheme 16 Reactions of trifluorodiazoethane with boronic acids.

Depending on the work-up conditions, Molander and coworkers obtained the gem-difluoro vinyl compound $\mathbf{7 4}$ after elimination of fluoride. Wu and co-workers took up this observation and developed a protocol that allows the conversion of aromatic boronic acids to either trifluoroethyl or difluorovinyl substituted aromatic compounds, depending on the reaction conditions (Scheme 16). ${ }^{61}$

\subsection{Lewis acid catalyzed transformations}

The homologation reaction of carbonyl compounds with diazo compounds is another very important application of this class of reagents. ${ }^{62,63}$ The Tiffeneau-Demjanov, ${ }^{64}$ Roskamp $^{65}$ and Buchner-Curtius-Schlotterbeck ${ }^{66,67}$ reactions are among the best-studied reactions in this context. Fluoroalkyl substituted diazo compounds possess outstanding potential for these transformations and allow the direct and facile introduction of fluoroalkyl groups to common organic building blocks.

Mock and co-workers reported in 1977 on the reaction of diazo compounds with ketones. ${ }^{68,69}$ Yet, the scope of this particularly useful transformation with trifluorodiazoethane was not investigated. In 1981, Wakselman and co-workers reported on Lewis-acid catalysed rearrangement reactions of the adduct of trifluorodiazoethane and aldehydes, yielding epoxides or ketones depending on the reaction conditions and the Lewis acid. ${ }^{21}$ In the presence of $\mathrm{BF}_{3} \cdot \mathrm{Et}_{2} \mathrm{O}$ the rearrangement of benzaldehyde to the corresponding homologous fluorinated hydratropic aldehyde was observed, while $\mathrm{SbCl}_{5}$ provided the epoxide. Although, this transformation is particularly useful for the synthesis of fluorinated ketones and epoxides, further investigations regarding the Tiffeneau-Demjanov and Roskamp reaction of carbonyl groups and trifluorodiazoethane were reported only recently by Carreira and co-workers.

Careful mechanistic investigations revealed $\mathrm{ZrCl}_{4}$ to be the best Lewis acid for the Roskamp reaction of aldehydes $\mathbf{7 5}$ and trifluorodiazoethane 1 to yield the corresponding ketones with high yield. Interestingly, in the case of aromatic aldehydes, Carreira and co-workers observed a reaction product that is generated after an initial aryl migration yielding hydratropic aldehyde 77, which undergoes a second addition-rearrangement reaction with trifluorodiazoethane to yield ketone $\mathbf{7 8}$. Notably, in this case the hydride migration is preferred (Scheme 17). ${ }^{70}$

Cyclic ketones $(\mathbf{8 0})$ react with trifluorodiazoethane in a Tiffeneau-Demjanov reaction affording $\alpha$-trifluoromethyl ringexpanded ketones $(\mathbf{8 1}) .^{70}$ In a similar way acetals $(\mathbf{8 2})$ can be

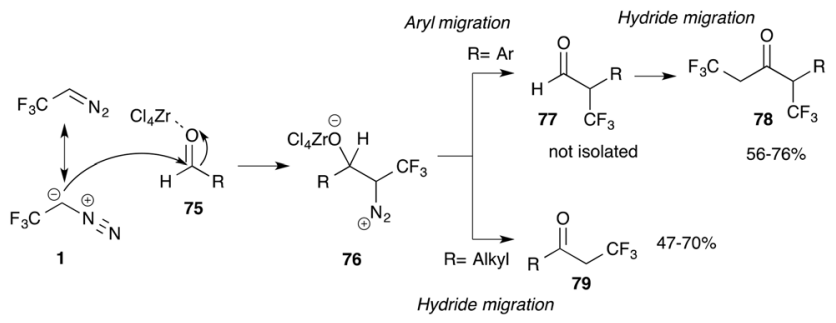

Scheme 17 Homologation of ketones.

homologated to the corresponding $\alpha$-branched acetals $(\mathbf{8 3})^{71}$ and imines (84) undergo reaction to yield the corresponding aziridines $(\mathbf{8 5}){ }^{72}$ Salicylaldehydes (86) provide the corresponding benzofuranols (49) (Scheme 18). ${ }^{50}$

Recently, Kappe and co-workers reported on the addition of trifluorodiazoethane to a range of different aldehydes and ketones using a continuous-flow protocol for the generation of trifluorodiazoethane. A tube-in-tube reactor was applied to transfer the diazo compound from the aqueous layer to the organic layer. Subsequent addition reaction of the stream of the diazo compound to a stream of the carbonyl compound in the presence of a base yielded the addition product in high yield and no subsequent rearrangement was observed. ${ }^{73}$

\subsection{Further transformation}

In the previous sections the reactivity of trifluorodiazoethane was discussed and different cycloaddition and nucleophilic addition reactions of this reagent have been discussed.

Recently, Mykhailiuk and co-workers reported on an unexpected transformation when using trifluorodiazoethane. Treatment of this diazo compound with nucleophiles such as $\beta$-ketoesters or other 1,3-dicarbonyl groups yields the addition product of the nucleophile to the terminal nitrogen of the diazo compound, that is hydrazone 89. In the presence of an

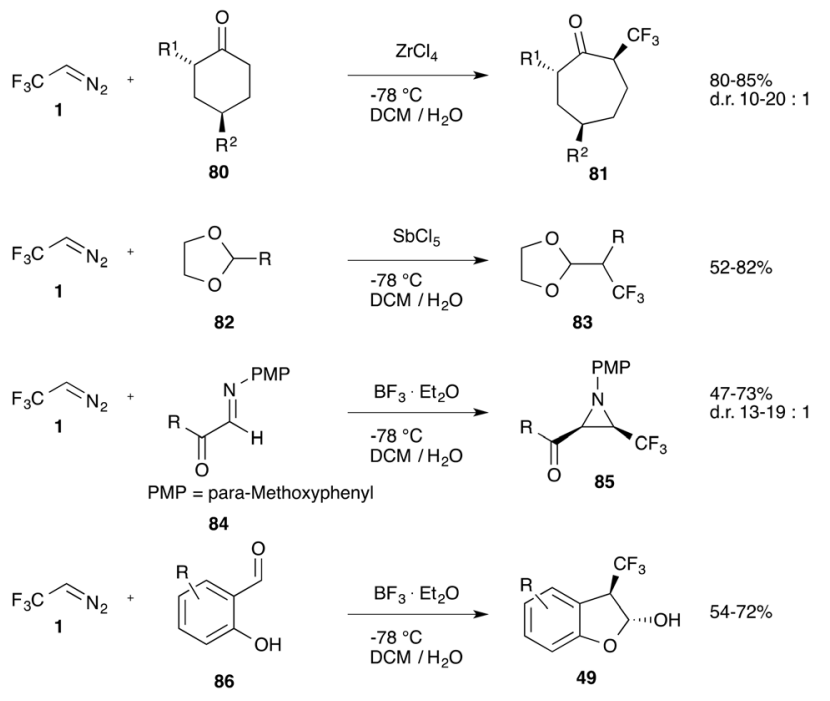

Scheme 18 Reactions of different carbonyl compounds. 


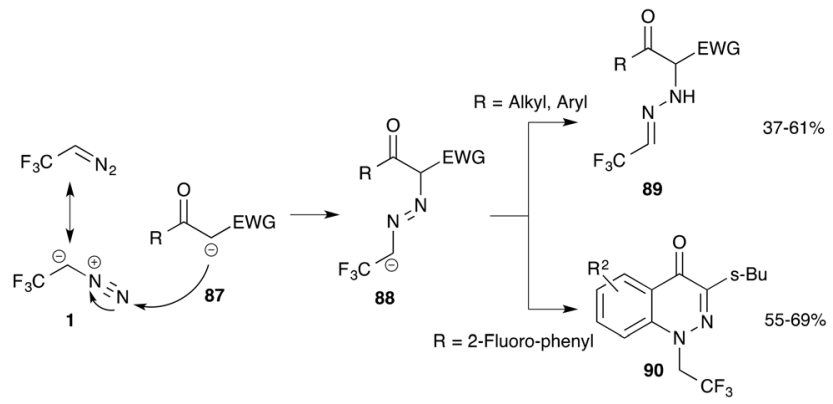

Scheme $19 \mathrm{~N}$-Terminal electrophilic reactivity of trifluorodiazoethane.

ortho-fluoro substituent in the proximity of the hydrazone, subsequent ring closure provides fluoroalkyl substituted cinnolinones (90). This observation is remarkable, as typically diazo compounds do react under extrusion of nitrogen and form a new bond at the diazo-carbon atom (Scheme 19). ${ }^{74}$

\section{Perfluorinated diazoalkanes}

While many applications on trifluorodiazoethane have been reported to date, longer chain perfluorinated diazoalkanes have been investigated to a much lower extent. Additionally, Fields and Haszeldine reported severe explosions when working with these diazo compounds. ${ }^{11}$ After the initial reports by Fields and co-workers synthetic applications of this class of diazo compounds have been scarce. Mykhailiuk was first to report on 2,2,3,3,3-pentafluorodiazopropane (3b) in 2014 and its application in dipolar cycloadditions yielding pyrazolines. ${ }^{75}$ Quickly after this report, Mykhailiuk showed that different short-perfluorinated diazo compounds (3a-b) can be prepared in batch and applied in the synthesis of pyrazolines using the one-pot biphasic protocol (Scheme 20). ${ }^{76}$

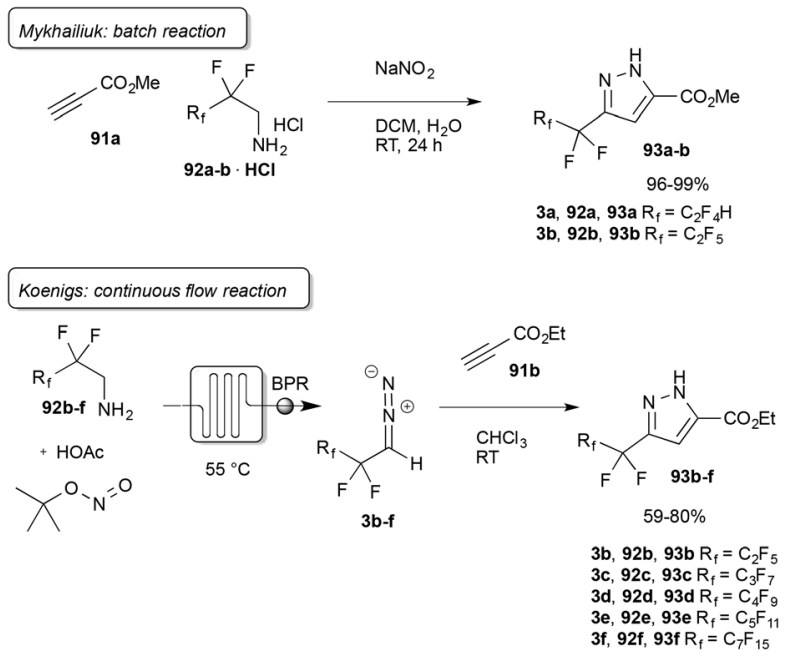

Scheme 20 Perfluorinated diazoalkanes reported by Mykhailiuk and Koenigs, BPR $=$ back pressure regulator.
Koenigs and co-workers further investigated the synthesis of perfluoroalkyl-substituted diazoalkanes (3b-f) in continuous-flow. A range of different perfluorinated amines was applied in a continuous-flow protocol using tert butyl nitrite and catalytic amounts of acetic acid for the preparation of perfluorinated diazoalkanes with different chain lengths (Scheme 20). ${ }^{47}$

\section{Difluorodiazoethane}

In the previous sections, synthetic applications of trifluorodiazoethane or longer-chain perfluorinated analogues have been described in detail. From a drug discovery point of perspective, the difluoromethyl group finds regular application as a substitute for the trifluoromethyl group. The advantage is that difluoromethyl is not as lipophilic as trifluoromethyl and it may interact as a hydrogen bond donor. Additionally, the difluoromethyl group is slightly protic, it can interact with a target protein as a hydrogen bond donor and thus finds regular application as an isostere of thiols and alcohols. ${ }^{77,78}$

Interestingly, difluorodiazoethane (2) was not reported in the literature until 2015, when Mykhailiuk reported the first synthesis and application in the synthesis of pyrazoles (95) in batch reactions. Mykhailiuk reported difluorodiazoethane to be highly sensitive to water and that the application of organic nitrites is crucial for its synthesis. ${ }^{79}$ Shortly after the initial report of Mykhailiuk, Koenigs and co-workers reported on the continuous-flow synthesis of difluorodiazoethane and its application to pyrazole and pyrazoline synthesis (96) (Scheme 21). ${ }^{47}$

\section{Summary and outlook}

After the first description of trifluorodiazoethane in 1943, this highly versatile reagent was applied only to a very limited extent in organic synthesis until it gained significant interest in the organic synthetic methodology. Over the past 6 years about 40 different publications have reported on novel
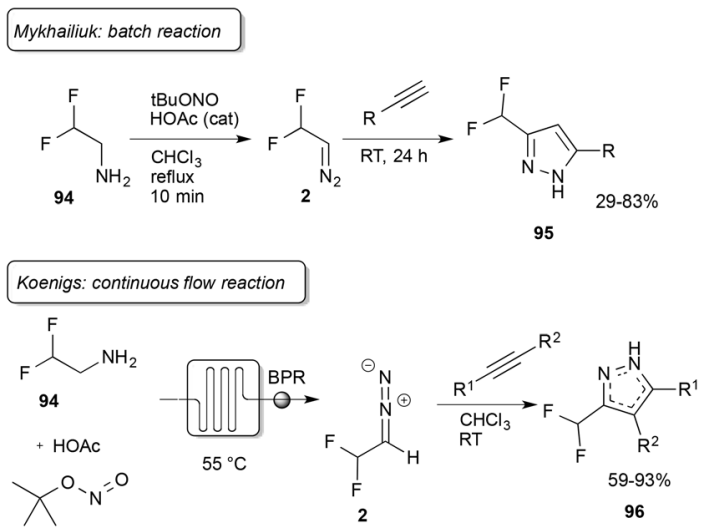

Scheme 21 Reactions with difluorodiazoethane. 
synthetic procedures for fluorinated building blocks, which are of fundamental interest to chemists, ranging from drug discovery to materials sciences. The recent advent of synthetic applications of this small and valuable reagent clearly outlines that in the next years this reagent will find its way into standard organic synthesis protocols that can be utilized, from both an academic and an industrial perspective. These new protocols do not require the hazardous isolation of this special reagent and rely on phase-transfer reactions, continuous-flow chemistry and simple one-pot protocols and enables chemists to better understand reactivity and to find novel and interesting reactivities. Noteworthily, this reagent does not react with carboxylic acids under the formation of an ester and it can react as an electrophile at the diazo group terminal nitrogen, underlining the unique chemical versatility of this reagent.

Recently, the corresponding difluoromethyl substituted analogue was reported and new reactions with this reagent are of current interest to organic chemists as it opens up new avenues to difluoromethyl-substituted building blocks.

\section{References}

1 C. B. H. von Pechmann, Chem. Ber., 1894, 27, 1888-1891.

2 A. Ford, H. Miel, A. Ring, C. N. Slattery, A. R. Maguire and M. A. McKervey, Chem. Rev., 2015, 115, 9981-10090.

3 T. Ye and M. A. McKervey, Chem. Rev., 1994, 94, 1091-1160.

4 S. T. R. Müller and T. Wirth, ChemSusChem, 2015, 2, 245250.

5 R. L. Hartman, J. P. McMullen and K. F. Jensen, Angew. Chem., Int. Ed., 2011, 50, 7502-7519.

6 B. J. Deadman, S. G. Collins and A. R. Maguire, Chem. Eur. J., 2015, 21, 2298-2308.

7 H. Gilman and R. G. Jones, J. Am. Chem. Soc., 1943, 65, 1458-1460.

8 M. A. Omary and I. W. H. Oswald, WO 2015084906, 2015.

9 S. Purser, P. R. Moore, S. Swallow and V. Gouverneur, Chem. Soc. Rev., 2008, 37, 320-330.

10 K. Müller, C. Faeh and F. Diederich, Science, 2007, 317, 1881-1886.

11 R. Fields and R. N. Haszeldine, J. Chem. Soc., 1964, 18811889.

12 J. H. Atherton and R. Fields, J. Chem. Soc. C, 1967, 14501454.

13 J. H. Atherton and R. Fields, J. Chem. Soc. C, 1968, 15071513.

14 J. H. Atherton and R. Fields, J. Chem. Soc. C, 1968, 22762278.

15 J. Cooke, W. R. Cullen, M. Green and F. G. A. Stone, Chem. Commun., 1968, 170-171.

16 C. O. Meese, Synthesis, 1984, 1041-1042.

17 N. A. Karst, T. F. Islam and R. J. Linhardt, Org. Lett., 2003, $5,4839-4842$.

18 D. T. Loehr, D. Armistead, J. Roy and H. C. Dorn, J. Fluorine Chem., 1988, 39, 283-287.
19 C. Wakselman and M. Tordeux, J. Fluorine Chem., 1982, 21, 99-106.

20 R. Fields and J. F. Tomlinson, J. Fluorine Chem., 1979, 13, 19-28.

21 M. Tordeux and C. Wakselman, J. Fluorine Chem., 1981, 17, 299-304.

22 R. Fields and J. P. Tomlinson, J. Fluorine Chem., 1979, 13, 147-158.

23 H.-D. Martin, R. Iden, F.-J. Mais, G. Kleefeld, A. Steigel, B. Fuhr, O. Rümmele, A. Oftring and E. Schwichtenberg, Tetrahedron Lett., 1983, 24, 5469-5472.

24 H. Lebel, J. F. Marcoux, C. Molinaro and A. B. Charette, Chem. Rev., 2003, 103, 977-1050.

25 J. Hansen and H. M. L. Davies, Coord. Chem. Rev., 2008, 252, 545-555.

26 H. M. L. Davies and J. R. Denton, Chem. Soc. Rev., 2009, 38, 3061-3071.

27 P. Le Maux, S. Juillard and G. Simonneaux, Synthesis, 2006, 1701-1704.

28 J. R. Denton, D. Sukumaran and H. M. L. Davies, Org. Lett., 2007, 9, 2625-2628.

29 P. K. Mykhailiuk, S. Afonin, A. S. Ulrich and I. V. Komarov, Synthesis, 2008, 1757-1760.

30 P. K. Mykhailiuk, S. Afonin, G. V. Palamarchuk, O. V. Shishkin, A. S. Ulrich and I. V. Komarov, Angew. Chem., Int. Ed., 2008, 47, 5765-5767.

31 B. Morandi and E. M. Carreira, Angew. Chem., Int. Ed., 2010, 49, 938-941.

32 B. Morandi, J. Cheang and E. M. Carreira, Org. Lett., 2011, 13, 3080-3081.

33 B. Morandi, B. Mariampillai and E. M. Carreira, Angew. Chem., Int. Ed., 2011, 50, 1101-1104.

34 B. Morandi and E. M. Carreira, Angew. Chem., Int. Ed., 2010, 49, 4294-4296.

35 O. S. Artamonov, E. Y. Slobodyanyuk, D. M. Volochnyuk, I. V. Komarov, A. A. Tomachev and P. K. Mykhailiuk, Eur. J. Org. Chem., 2014, 3592-3598.

36 O. S. Artamonov, E. Y. Slobodyanyuk, O. V. Shishkin, I. V. Komarov and P. K. Mykhailiuk, Synthesis, 2013, 225230.

37 T. R. Li, S. W. Duan, W. Ding, Y. Y. Liu, J. R. Chen, L. Q. Lu and W. J. Xiao, J. Org. Chem., 2014, 79, 2296-2302.

38 C.-L. Zhu, L.-J. Yang, S. Li, Y. Zheng and J.-A. Ma, Org. Lett., 2015, 17, 3442-3445.

39 C. L. Zhu, J. A. Ma and D. Cahard, Asian J. Org. Chem., 2016, 5, 66-69.

40 M. A. J. Duncton, L. Ayala, C. Kaub, S. Janagani, W. T. Edwards, N. Orike, K. Ramamoorthy, J. Kincaid and M. G. Kelly, Tetrahedron Lett., 2010, 51, 1009-1011.

41 A. Padwa, 1,3-Dipolar Cycloaddition Chemistry, John Wiley \& Sons, New York, 1984.

42 T. D. Penning, J. J. Talley, S. R. Bertenshaw, J. S. Carter, P. W. Collins, S. Doctor, M. J. Graneto, L. F. Lee, J. W. Malecha, J. M. Miyashiro, R. S. Rogers, D. J. Rogier, S. S. Yu, G. D. Anderson, E. G. Burton, J. N. Cogburn, S. A. Gregory, C. M. Koboldt, W. E. Perkins, K. Seibert, 
A. W. Veenhuizen, Y. Y. Zhang and P. C. Isakson, J. Med. Chem., 1997, 40, 1347-1365.

43 S. R. Bertenshaw, J. S. Carter, P. W. Collins, S. H. Doctor, M. J. Granets, I. K. Khanna, J. W. Malecha, J. M. Miyashiro, T. D. Penning, R. S. Rogers, D. J. Rogier Jr., J. J. Talley and and S. S. Yu, WO 9515316, 1995.

44 M. L. Quan, P. Y. S. Lam, Q. Han, D. J. P. Pinto, M. Y. He, R. Li, C. D. Ellis, C. G. Clark, C. A. Teleha, J.-H. Sun, R. S. Alexander, S. Bai, J. M. Luettgen, R. M. Knabb, P. C. Wong and R. R. Wexler, J. Med. Chem., 2005, 48, 1729-1744.

45 F. Li, J. Nie, L. Sun, Y. Zheng and J. A. Ma, Angew. Chem., Int. Ed., 2013, 52, 6255-6258.

46 E. Y. Slobodyanyuk, O. S. Artamonov, O. V. Shishkin and P. K. Mykhailiuk, Eur. J. Org. Chem., 2014, 2487-2495.

47 L. Mertens, K. J. Hock and R. M. Koenigs, Chem. - Eur. J., 2016, 22, 9542-9545.

48 F.-G. Zhang, Y. Wei, Y.-P. Yi, J. Nie and J.-A. Ma, Org. Lett., 2014, 16, 3122-3125.

49 L. Sun, J. Nie, Y. Zheng and J. A. Ma, J. Fluorine Chem., 2015, 174, 88-94.

50 B. Morandi and E. M. Carreira, Org. Lett., 2011, 13, 59845985.

51 G. A. Molander and L. N. Cavalcanti, Org. Lett., 2013, 15, 3166-3169.

52 A.-J. Cai, Y. Zhenga and J.-A. Ma, Chem. Commun., 2015, 51, 8946-8949.

53 H. M. L. Davies and D. Morton, Chem. Soc. Rev., 2011, 40, 1857-1869.

54 H. M. L. Davies and S. J. Hedley, Chem. Soc. Rev., 2007, 36, 1109-1119.

55 D. Gillingham and N. Fei, Chem. Soc. Rev., 2013, 42, 49184931.

56 C.-B. Liu, W. Meng, F. Li, S. Wang, J. Nie and J.-A. Ma, Angew. Chem., Int. Ed., 2012, 51, 6227-6230.

57 H. Q. Luo, G. J. Wu, Y. Zhang and J. B. Wang, Angew. Chem., Int. Ed., 2015, 54, 14503-14507.

58 S. Hyde, J. Veliks, B. Liegault, D. Grassi, M. Taillefer and V. Gouverneur, Angew. Chem., Int. Ed., 2016, 55, 3785-3789.
59 O. A. Argintaru, D. Ryu, I. Aron and G. A. Molander, Angew. Chem., Int. Ed., 2013, 52, 13656-13660.

60 G. A. Molander and D. Ryu, Angew. Chem., Int. Ed., 2014, 53, 14181-14185.

61 G. J. Wu, Y. F. Deng, C. Q. Wu, X. Wang, Y. Zhang and J. B. Wang, Eur. J. Org. Chem., 2014, 4477-4481.

62 W. Kirmse, Eur. J. Org. Chem., 2002, 2193-2256.

63 N. R. Candeias, R. Paterna and P. M. P. Gois, Chem. Rev., 2016, 116, 2937-2981.

64 P. W. Marc Tiffeneau and B. Tchoubar, Comptes Rendus, 1937, 205, 54-56.

65 C. R. Holmquist and E. J. Roskamp, J. Org. Chem., 1989, 54, 3258-3260.

66 E. Buchner and T. Curtius, Ber. Dtsch. Chem. Ges., 1885, 18, 2371-2377.

67 F. Schlotterbeck, Ber. Dtsch. Chem. Ges., 1826, 40, 18261827.

68 W. L. Mock and M. E. Hartman, J. Org. Chem., 1977, 42, 466-472.

69 W. L. Mock and M. E. Hartman, J. Org. Chem., 1977, 42, 459-465.

70 B. Morandi and E. M. Carreira, Angew. Chem., Int. Ed., 2011, 50, 9085-9088.

71 J. Y. Hamilton, B. Morandi and E. M. Carreira, Synthesis, 2013, 1857-1862.

72 S. A. Kunzi, B. Morandi and E. M. Carreira, Org. Lett., 2012, 14, 1900-1901.

73 B. Pieber and C. O. Kappe, Org. Lett., 2016, 18, 1076-1079.

74 A. V. Arkhipov, V. V. Arkhipov, J. Cossy, V. O. Kovtunenko and P. K. Mykhailiuk, Org. Lett., 2016, 18, 3406-3409.

75 P. K. Mykhailiuk, Chem. - Eur. J., 2014, 20, 4942-4947.

76 P. K. Mykhailiuk, Org. Biomol. Chem., 2015, 13, 3438-3445.

77 J. Hu, W. Zhang and F. Wang, Chem. Commun., 2009, 74657478.

78 J. Hu and C. Ni, Sci. Synth., C-1 Build. Blocks Org. Synth., 2014, 409-458.

79 P. K. Mykhailiuk, Angew. Chem., Int. Ed., 2015, 54, 65586561. 